\title{
Cup anemometer measurement errors due to problems in the output signal generator system
}

\author{
Alvaro Ramos-Cenzano, Mikel Ogueta-Gutiérrez, Santiago Pindado* \\ Instituto Universitario de Microgravedad "Ignacio Da Riva” (IDR/UPM), ETSI Aeronáutica y del Espacio, Universidad Politécnica de Madrid, Pza. del Cardenal Cisneros 3, Madrid, 28040, Spain
}

\section{A R T ICLE INFO}

\section{Keywords}

Cup anemometer

Anemometer calibration

Output frequency

FFT

Thies first class advanced

Maintenance

\begin{abstract}
A B S T R A C T
The effect of drops in the rotation wheel of the opto-electronic system of a commercial anemometer (Thies First Class Advanced) was studied. The output voltage of this anemometer was measured at different wind speeds during its calibration process, at $25 \mathrm{kHz}$ sampling frequency along $25 \mathrm{~s}$. The output frequency was calculated both ways: by counting pulses (CP) and by carrying out a Fast Fourier Transform (FFT). The errors of the anemometers transfer function based on both procedures are given in relation to the official (MEASNET) calibration. The effect of the sampling frequency is also analyzed together with the presence of oil drops. No effect of the oil drops was observed on calibrations carried out by extracting the anemometers transfer function with FFT. When using CP, results showed great differences between calibrations affected and not affected by the presence of oil drops in the rotation wheel of the opto-electronic system.
\end{abstract}

\section{Introduction}

The frequency of the output signal of a cup anemometer, usually called output frequency, $f$, is the parameter used to calculate the measured wind velocity, no matter if this output signal is a sine wave generated by a magnets system or a square wave generated by an opto-electronic system.

Bearing in mind that normally the output signal from the most common top-class anemometers (Thies First Class Advanced or Vector Instruments A100 LK/LM) is a square wave signal composed by a quite large number of pulses per turn, it is important to sample it adequately in order to extract an accurate value of the aforementioned output frequency.

Anemometer degradation is a well-known problem in the wind energy sector as well as in meteorology. As a cup anemometer loses performance due to:

-the normal wear and tear process,

-an incorrect maintenance, or

•sudden incidents such as lightning,

the wind speed measurement given by the instrument diverges from the real wind speed. As a consequence, this loss of performance is translated into a wrong wind turbine operation or inaccurate data when studying the energy production of a specific geographic location, causing a negative impact on the foreseen industrial revenue in both cases. This isn't a new problem neither in the wind energy sector nor in the meteorology one. Furthermore, it can be said that around 30\% of mast-mounted anemometers return for recalibration far from normal operational conditions [1].

In order to preserve its performance, cup anemometers need a thorough and periodical maintenance to keep the lowest possible friction between the bearings, this maintenance including a proper lubrication. However, lubrication can cause problems in the opto-electronic system. In Fig. 1, the presence of an oil drop at the slotted wheel of the opto-electronic system from a Thies First Class Advanced cup anemometer is shown. These oil drops can alter the output signal of the anemometer, as the output signal might lose pulses in each turn.

The output signal of a cup anemometer is shown in Fig. 2, this signal being a square wave in which the higher voltage level corresponds to the moment when a slot of the wheel, see Fig. 1, lets the light generated by a led passing through it and, consequently, a light sensor located at the other side of the wheel is illuminated. In this figure, the open circles represent a sampling data obtained at $830 \mathrm{~Hz}$ rate for three different wind speeds (that imply three different rotation rates of the cup anemometer, obviously). It can be observed that at larger wind speeds it is necessary to increase the sampling rate if an accurate measurement of the anemometer's rotation rate is required.

\footnotetext{
* Corresponding author.

Email address: santiago.pindado@upm.es (S. Pindado)
} 


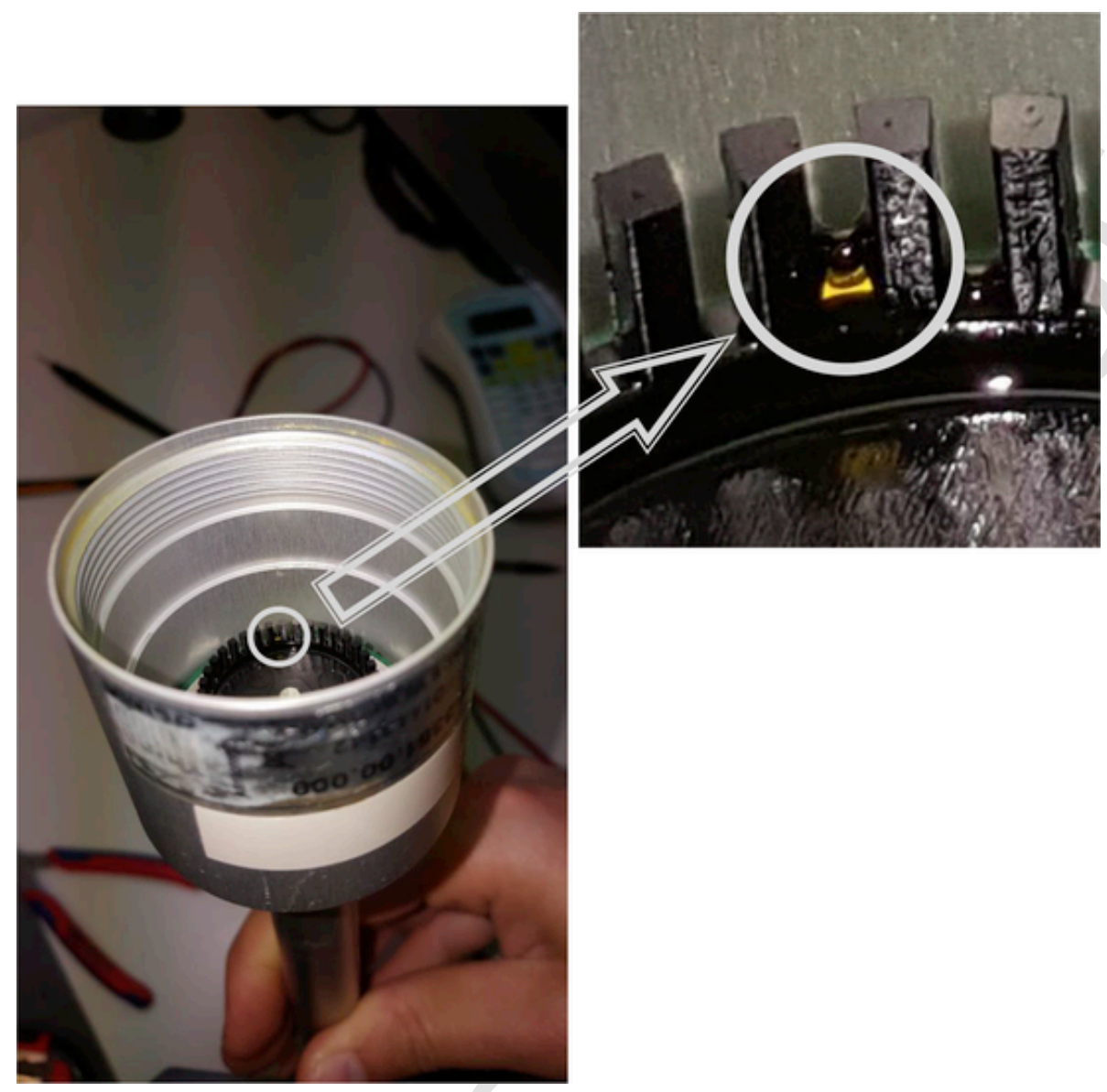

Fig. 1. (Left) Oil drop in the slotted wheel of the optoelectronic system's of a Thies First Class Advanced cup anemometer. (Right) Fluid meniscus of the oil drop.

The aforementioned effect of oil drops in the slotted wheel was already checked at the IDR/UPM Institute, together with its surprising, though logical, disappearance at the larger wind speeds (at higher rotation rates of the slotted wheel, the fluid meniscus of the drop tends to vanish due to the effect of the inertia combined with the surface tension of the drop).

In this work, the effect of the presence of drops in the opto-electronic system of a Thies First Class Advanced cup anemometer is analyzed. The oil drops have been simulated in the output signals records of this anemometer taken during its calibration. In every lap, pulses of the signal were eliminated by changing their higher voltage levels by the lower ones. This is shown in Fig. 3, where a time series of the voltage level, $e$, sampled from a cup anemometer's signal can be observed, together with a time series where a drop at on slot has been simulated by deleting one pulse. Besides, two methods for extracting the output frequency from a sampled square wave signal, Counting Pulses (CP) and the Fast Fourier Transform (FFT), are compared. The effect of the sampling frequency is also studied.

This paper is organized as follows. In Section 2 the calibration and post-processing process are described, together with the studied cases. The results are discussed in Section 3, and the conclusions are finally summarized in Section 4.

\section{Testing configuration and cases studied}

The cup anemometer performance is commonly evaluated through the transfer function:

$V=A \cdot f+B$,

that gives the wind speed, $V$, as a function of the output frequency of the anemometer, $f$. The slope and offset (A and B, respectively) of the above equation have to be defined by a proper calibration process. The data from the calibration of a Thies Clima First-Class Advanced anemometer is analyzed in this work. This calibration was performed at the LAC-IDR/UPM calibration lab, following the procedure stated by MEASNET. More information on this facility and the lab can be found in Refs. [3-7].

In brief, a cup anemometer calibration is a wind-tunnel testing of this sensor in which a correlation between the output frequency of the instrument and the wind-flow speed is carried out. Thirteen measurement points are taken between $4 \mathrm{~m} \mathrm{~s}^{-1}$ and $16 \mathrm{~m} \mathrm{~s}^{-1}$ to stablish that correlation, and to define the aforementioned transfer function by extracting the constants A and B through a linear fitting of the data. The proper transfer function, obtained for the studied cup anemometer by following MEASNET standards during their calibration process $[8,9]$, is taken as the reference data calibration (hereinafter defined by constants $A^{*}$ and $\mathrm{B}^{*}$, see equation (1)) in the present work.

During each point of this calibration, a 25-s sample of the anemometer output signal was recorded at $25 \mathrm{kHz}$ with a National Instruments NI USB-6210 Data Acquisition System. New data sets with different sampling frequencies were generated by extracting selected points from each data sample (e.g., the $1 \mathrm{kHz}$ samples were obtained taking one out of twenty-five points from the original samples). Therefore, 20 different calibration datasets of 13 wind speed points, at different sampling frequencies were generated, see Table 1 . In all those time series, one every 37 pulses has been modified in order to simulate the presence of an oil drop.

The aim of the present work is to study the effect of an oil drop in the slotted wheel of a cup anemometer on its transfer function accu- 


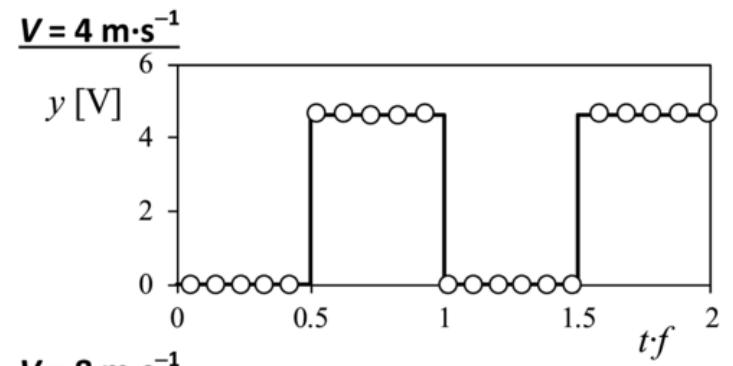

$V=8 \mathrm{~m} \cdot \mathrm{s}^{-1}$

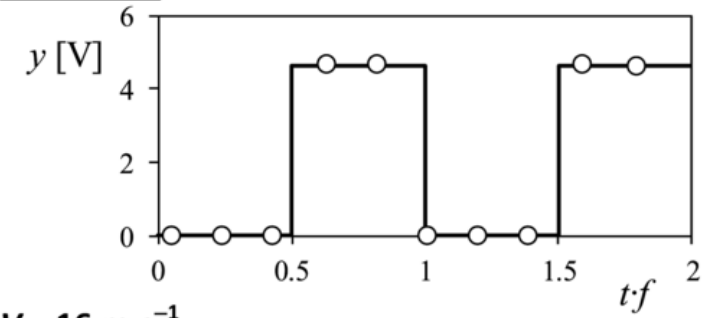

$\underline{V}=16 \mathrm{~m} \cdot \mathrm{s}^{-1}$

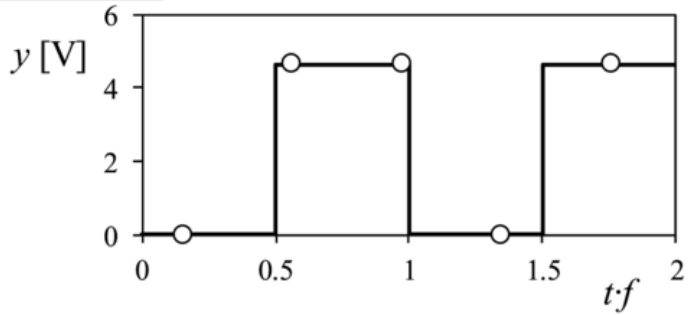

Fig. 2. Sampling data (open circles) extracted at $830 \mathrm{~Hz}$ rate from the output signal (square wave) of a cup anemometer, at three different wind speeds. In the figure $f$ stands for the frequency of the signal (also called the cup anemometer's output frequency, see equation (1)). From Ref. [2].

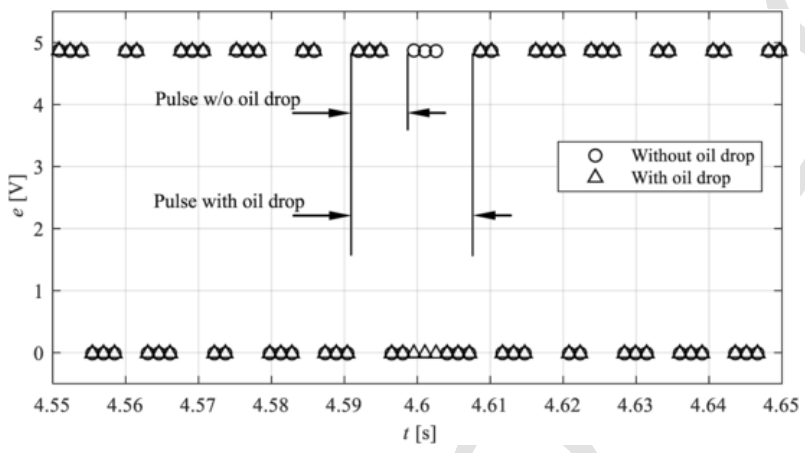

Fig. 3. Example of one pulse loss within one of the cup anemometer output signal datasets.

racy, taking into account: 1 ) the effects of the sampling frequency of the output signal, and 2) the way to extract the output frequency (comparing $\mathrm{CP}$ and FFT). The results are directly compared to the reference calibration of the anemometer, in order to quantify the aforementioned loss of accuracy.

Additionally, the presence of a second drop in the slotted wheel is also analyzed, as more than one drop might remain in the slotted wheel of the anemometer output system due to a sloppy maintenance. In this case, the position of the second drop in relation to the first one is relevant. Therefore, all possible cases are taken into account.

\section{Results}

In Fig. 4, the data (i.e., the transfer functions) from the $1000 \mathrm{~Hz}$ sampling rate datasets corresponding to:
Table 1

Cases analyzed in the present work.

\begin{tabular}{lll}
\hline Measurement points per calibration dataset & $\bullet 4 \mathrm{~m} \mathrm{~s}^{-1}$ & $\bullet 11 \mathrm{~m} \mathrm{~s}^{-1}$ \\
& $\bullet 5 \mathrm{~m} \mathrm{~s}^{-1}$ & $\bullet 12 \mathrm{~m} \mathrm{~s}^{-1}$ \\
$\bullet$ & $\bullet \mathrm{m} \mathrm{s}^{-1}$ & $\bullet 13 \mathrm{~m} \mathrm{~s}^{-1}$ \\
& $\bullet-8 \mathrm{~m} \mathrm{~s}^{-1}$ & $\bullet 14 \mathrm{~m} \mathrm{~s}^{-1}$ \\
& $\bullet 9 \mathrm{~m} \mathrm{~s}^{-1}$ & $\bullet 16 \mathrm{~m} \mathrm{~s}^{-1}$ \\
Length of the sample at each point & $\bullet 10 \mathrm{~m} \mathrm{~s}^{-1}$ & \\
Sampling frequencies analyzed & $\bullet 25 \mathrm{~s}$ & \\
& $\bullet 500 \mathrm{~Hz}$ & $\bullet 833.33 \mathrm{~Hz}$ \\
& $\bullet 625 \mathrm{~Hz}$ & $\bullet 862.07 \mathrm{~Hz}$ \\
& $\bullet 657.89 \mathrm{~Hz}$ & $\bullet 892.86 \mathrm{~Hz}$ \\
& $\bullet 675.98 \mathrm{~Hz}$ & $\bullet 925.93 \mathrm{~Hz}$ \\
& $\bullet 694.44 \mathrm{~Hz}$ & $\bullet 961.54 \mathrm{~Hz}$ \\
& $\bullet 714.29 \mathrm{~Hz}$ & $\bullet 1000 \mathrm{~Hz}$ \\
& $\bullet 735.29 \mathrm{~Hz}$ & $\bullet 1250 \mathrm{~Hz}$ \\
& $\bullet 757.58 \mathrm{~Hz}$ & $\bullet 5000 \mathrm{~Hz}$ \\
& $\bullet 781.25 \mathrm{~Hz}$ & $\bullet 12500 \mathrm{~Hz}$ \\
& $\bullet 806.45 \mathrm{~Hz}$ & $\bullet 25000 \mathrm{~Hz}$ \\
& $\bullet \mathrm{CP}$ & \\
$\bullet$ & $\bullet \mathrm{FFT}$ &
\end{tabular}

-the case with one drop in the slotted wheel of the anemometer, and -the case without any drop,

are shown together with the calibration curve from the proper process at IDR/UPM Institute (that is, the reference calibration curve). The output frequencies in each point were calculated by CP. In the graph it can be observed that the presence of the drop causes a lack of accuracy in relation to the output frequency, $f$, which would be translated into an incorrect calculation of the calibration constants, the slope, A, and the offset, $\mathrm{B}$, of the corresponding transfer function.

The accuracy of the calibration curves extracted from each dataset studied in the present work can be estimated in terms of the residuals:

$r_{s, i}=V_{i}-\left(A \cdot f_{i}+\mathrm{B}\right)$,

and the error of the wind speed with respect to the one resulting from the reference calibration curve [10]:

$\Delta V_{i}=\left(\mathrm{A}^{*} \cdot f_{i}+\mathrm{B}^{*}\right)-\left(\mathrm{A} \cdot f_{i}+\mathrm{B}\right)$

An example of the variation of the calibration residuals, $\left|r_{s}\right|$, with the velocity in the wind tunnel is shown for two different sampling rates ( $f_{1}=1000 \mathrm{~Hz}$ and $f_{2}=658 \mathrm{~Hz}$ ) in Fig. 5 . In the upper graph of this figure the results obtained with the CP method for extracting the output frequency of the anemometer are shown, whereas the results obtained by using the FFT method are respectively plotted in the lower graph. It can be observed in the figure that, for the selected datasets, the presence or absence of the oil drop does not significantly change the behavior of the residuals. As expected, lower values of the residuals are obtained (with both methods, CP and FFT) from the $1000 \mathrm{~Hz}$ dataset than from the $685 \mathrm{~Hz}$ dataset.

The corresponding variations of the velocity errors, $|\Delta V|$, are shown in Fig. 5. In this case, the results obtained with the FFT method show no remarkable differences between the calibration calculated from the dataset with the oil drop and the one calculated from the dataset without it, regardless of the sampling frequency. However, the influence of the oil drop on the calibrations obtained with the CP method is quite noticeable for both sampling rates. The presence of the oil drop dramatically increases the error in the measured wind speed. It is important to remark that the vertical scale of the graphs in Fig. 6 is logarithmic. Finally, it should also be highlighted that the lower error in relation to 


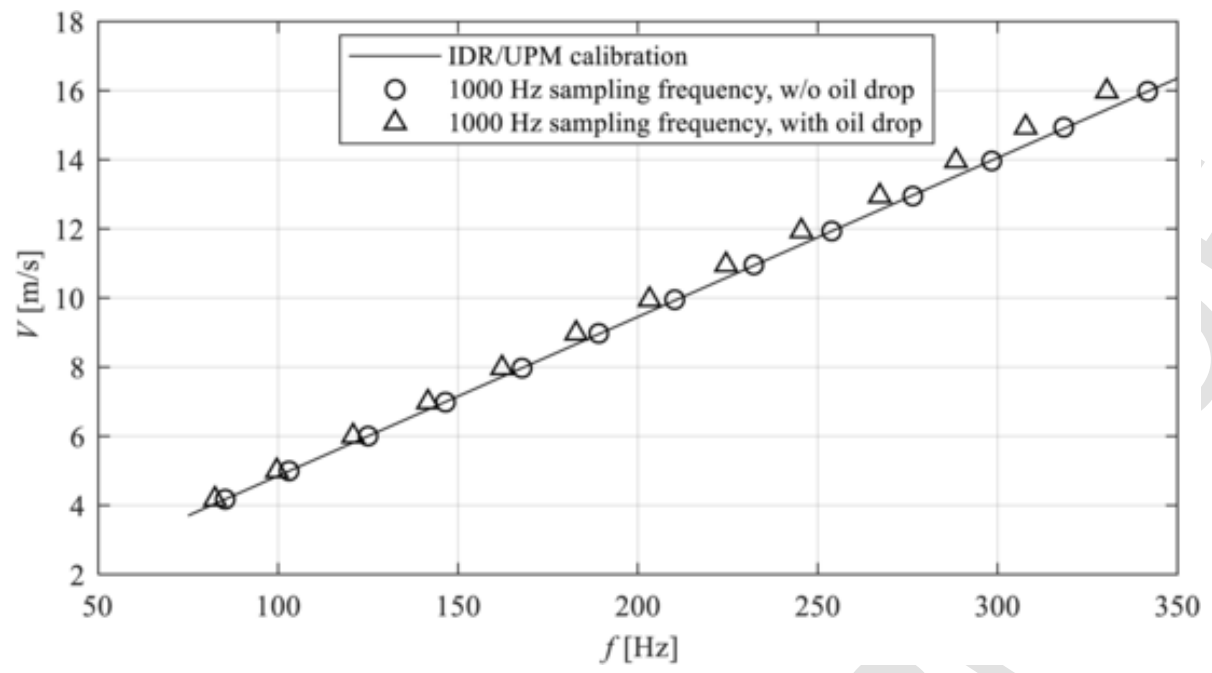

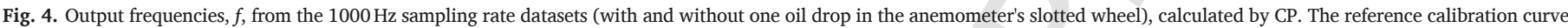
(IDR/UPM calibration) has been also included in the graph.
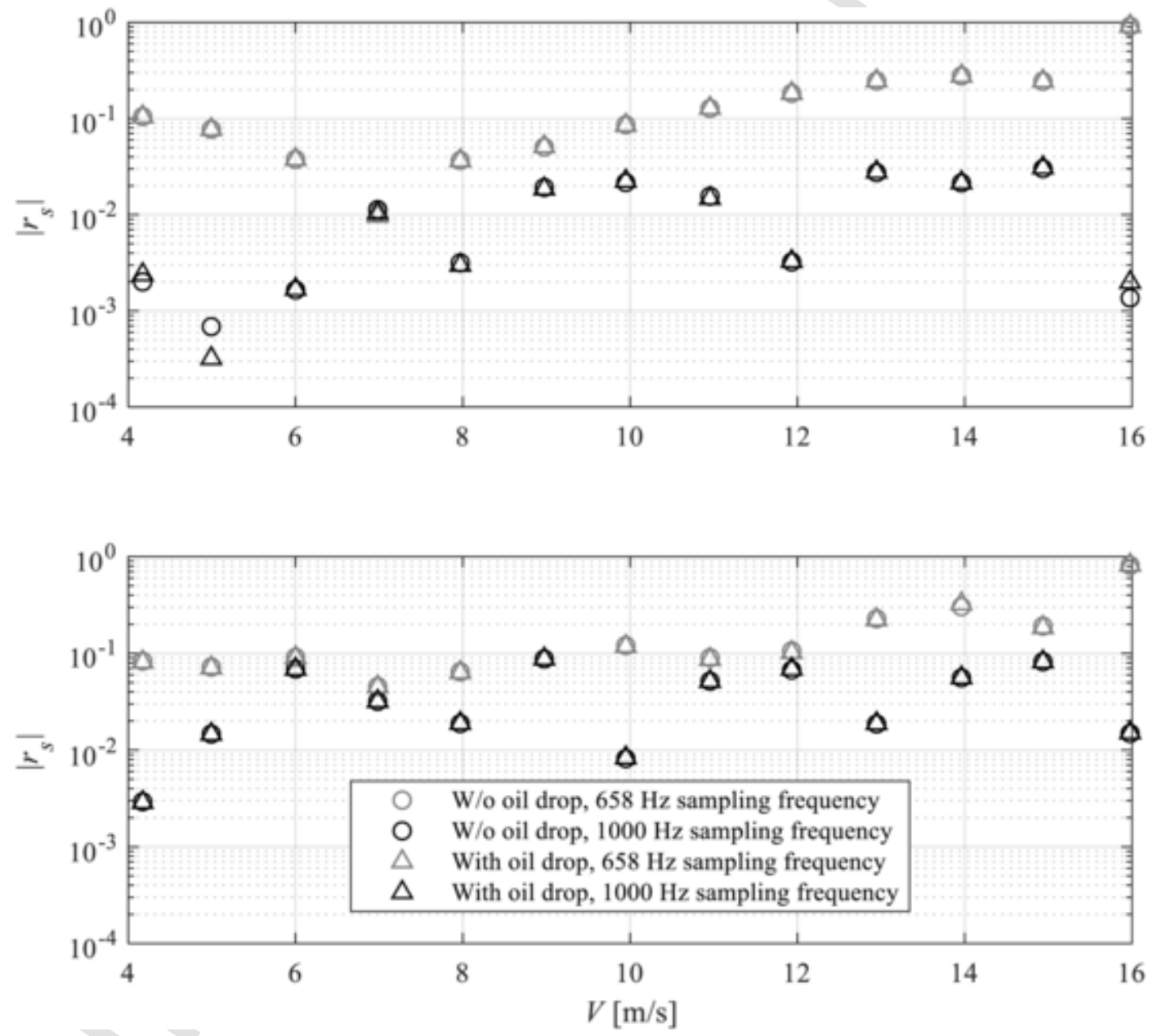

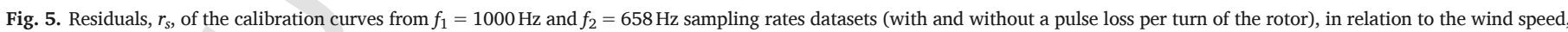
$V$. Results obtained by using CP (top graph) and FFT (bottom graph) procedures to extract the output frequencies of the anemometer.

the proper calibration of the cup anemometer is obtained by using CP (obviously, in the case of no oil drop in the slotted wheel of the wind sensor). This result was obtained in our previous work [10].

Additionally, an averaged value of the residuals, $R_{S}$, and an averaged value of the wind speed error, $\Delta V_{\text {avg: }}$ : 

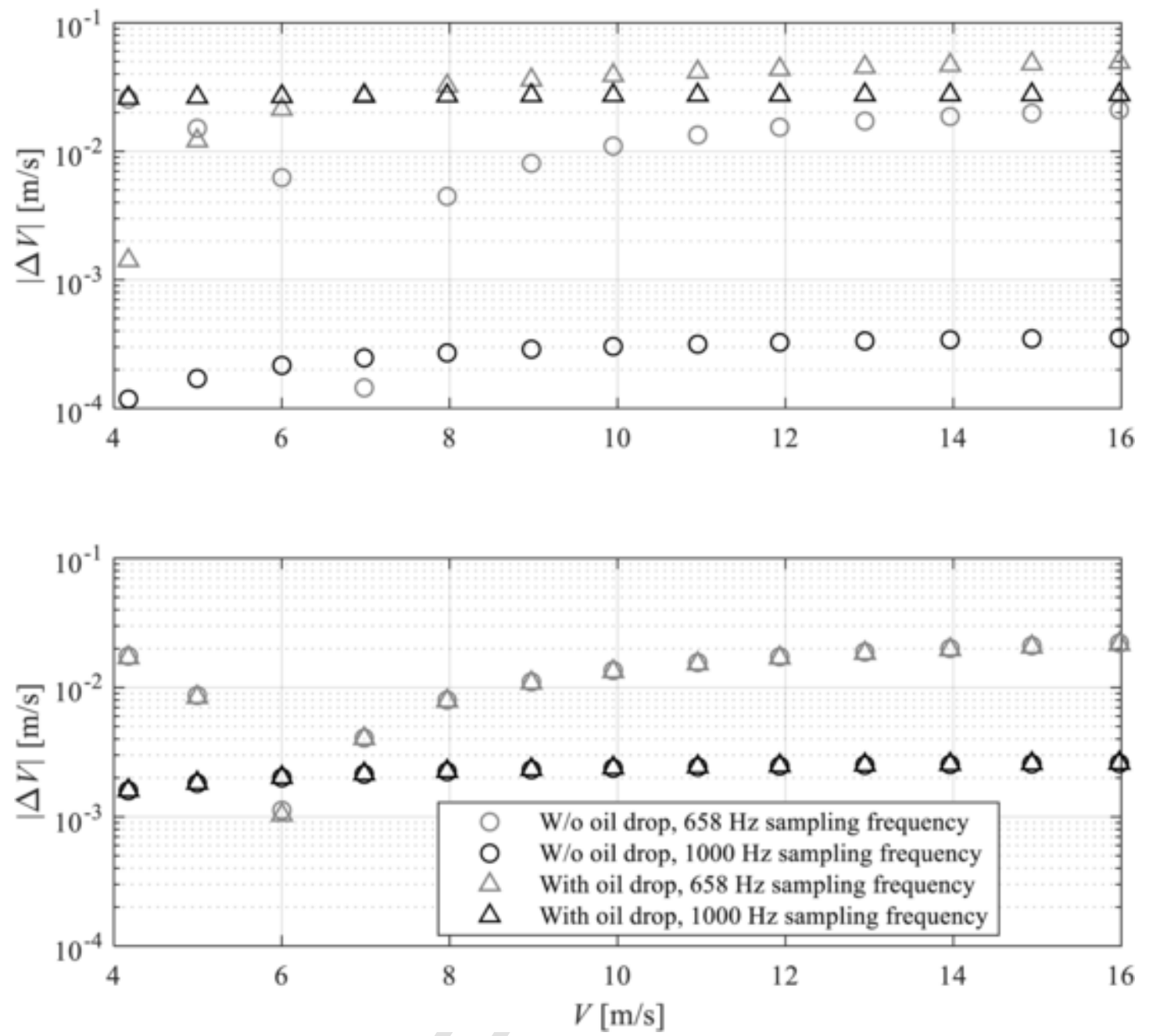

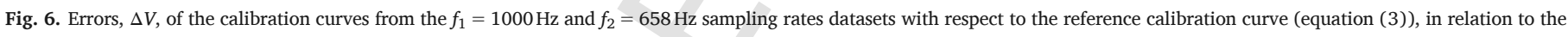
wind speed, $V$. Results obtained by using CP (top graph) and FFT (bottom graph) procedures to extract the output frequencies of the anemometer.

$\mathrm{R}_{s}=\frac{1}{13} \sum_{i=1}^{13}\left|r_{s, i}\right|$

$\Delta V_{a v g}=\frac{1}{13} \sum_{i-1}^{13} \frac{\left|\Delta V_{i}\right|}{\left(\mathrm{A}^{*} f_{i}+\mathrm{B}^{*}\right.}$,

have been calculated to characterize the accuracy of the transfer functions corresponding to all analyzed different sampling rate datasets. The values of these new variables in relation to the sampling rates up to $1000 \mathrm{~Hz}$ (the variables remain almost constant for larger frequencies) are shown in Figs. 7 and 8, respectively. From these figures it seems clear that the residuals of the calculated calibration curves are not affected by the presence of the oil drop, regardless the sampling frequency and the processing method, CP or FFT.

The explanation of this behavior in case of the calibrations based on $\mathrm{CP}$ lies on the definition of the residuals, which are the average differences between points and their linear fittings. The presence of the oil drop changes the points by introducing an error that is linear with the output frequency, as it can be observed in Fig. 4. Let's evaluate one point of a calibration (without drop) where $N_{i}$ pulses have been generated. Therefore, the output frequency can be calculated as:

$f_{i}=\frac{N_{i}}{\Delta t}$

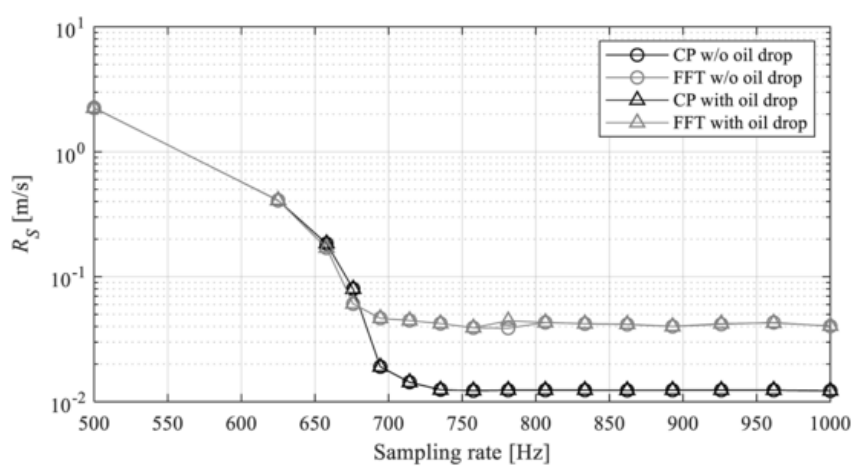

Fig. 7. Average residual, $R_{S}$ (see equation (4)), in relation to the sampling rate, calculated with both methods, CP and FFT, for the datasets with and without oil drop.

where $\Delta t=25 \mathrm{~s}$ is the length of the sample. On the other hand, the frequency corresponding to the oil drop case, $f_{i}^{*}$, can be estimated as:

$f_{i}^{*} \approx \frac{N_{i}-\frac{1}{37} N_{i}}{\Delta t}=\frac{36}{37} f_{i}$,

which means that the linearity of the calibrations from datasets affected by an oil drop remains when compared to the corresponding cal- 


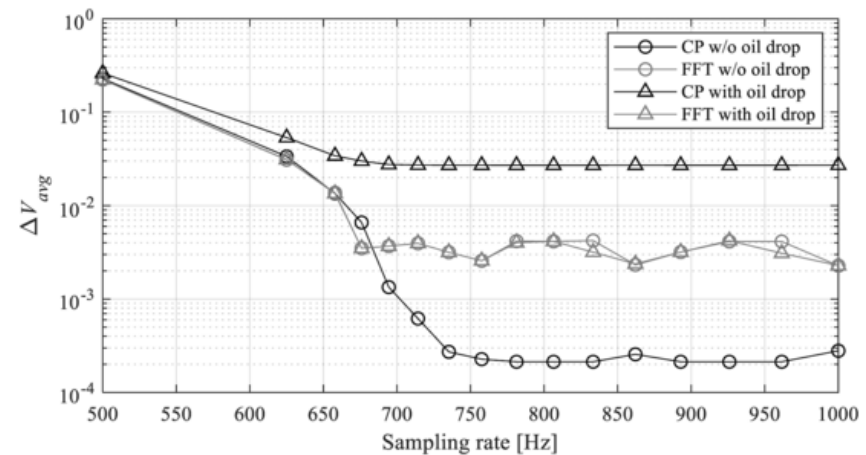

Fig. 8. Average error, $\Delta V_{\text {avg }}$ (see equation (5)), in relation to the sampling rate, calculated with both methods, CP and FFT, for the datasets with and without oil drop.

ibrations from datasets not affected by an oil drop. Therefore, it is logical to assume that the residuals should be very similar.

With regard to the calibrations based on FFT, the oil drop only introduces a perturbation each turn, whereas the time of the pulses generated by the slots of the slotted wheel remain the same in relation to the no-oil drop cases (the only difference is that 1 pulse out of 37is missing). Consequently, the calculated frequencies with FFT should be very similar when compared both cases with and without drop, the residuals being also very similar.

In contrast with the residuals, the wind speed error of the calibration curves is greatly affected by the presence of an oil drop in the anemometer's slotted wheel if the CP method is used for calculating the output frequency, $f$. The difference is of two orders of magnitude. As stated previously by equation (8), a percentage error is introduced in the calculations of the output frequency by $\mathrm{CP}$ when an oil drop is in the slotted wheel. The result is a calibration curve different from the reference one, which logically increases the wind speed error. In other words, the measurement of the velocity with the $\mathrm{CP}$ method when an oil drop accidently falls into a slot of the slotted wheel of the anemometers output system, can lead to a quite relevant differences with regard to the true wind speed (which could be a problem in terms of economic revenue in the wind energy sector, as the extractable energy from wind generator is proportional to the third power of the wind speed).

Besides, the calibrations based on FFT do not reveal differences in terms of velocity error when comparing the cases with and without oil drop. The reason for this result was mentioned above. The lack of one pulse due to the presence of an oil drop in the slotted wheel only introduces a perturbation each turn, therefore the FFT analysis will only result in an increase of the spectrum peak related to the cups rotation frequency, the one related to the output frequency (caused by the pulses) being almost unchanged.

In Fig. 8, the more accurate results obtained in calibrations based on $\mathrm{CP}$ of datasets without oil drop when compared to the ones based on FFT is shown. This result, previously commented, might require a further explanation. The FFT is a powerful mathematical tool to analyze datasets, it extracts the most relevant frequencies of a dataset up to a limit that depends on the sampling frequency and the length of the sample. Also, the FFT is a technique that needs to split the sample in order to keep $2^{n}$ points only. Therefore, its accuracy is limited when compared to CP, whose error is in terms of extracted frequency is around the inverse of the number of pulses recorded by the sample, and does not add related to the length of the sample, the sampling frequency, or the numerical errors due to the algorithms used.

Additionally, the effect of the presence of a second oil drop in the slotted wheel of the opto-electronic system has been analyzed. In this case, not only the sampling frequency and the processing method, but also the position of the second oil drop in relation to the first one have been studied. The comparison of the residuals from the different cases tested:

-no drop in the slotted wheel,

-one drop in the slotted wheel,

-drops in two consecutive slots of the wheel,

-drops in two diametrically opposed slots of the wheel (that is, the second drop is located in the 18th slot after the first drop, as the Thies First Class Advanced wheel comprises 37 slots),

is shown in Fig. 9. In the top graph of this figure, the results from the calibrations based on the CP method are shown, whereas the results from calibrations based on the FFT method are shown in the bottom graph.

Regarding the CP method, it is clear that no difference is found between any of the cases tested, regardless the sampling frequency. Therefore, it can be said that the CP method is very robust concerning the residuals of the calibration. The FFT method has also quite good results, but when the measurement is finally stabilized, the results indicate higher values of the residuals than those from the CP method. However, it can be said that the FFT method is also quite robust concerning the residuals of the calibration.

Fig. 10 is organized following the same pattern from Fig. 8, but in this case the average error of the calibrations, $\Delta V_{a v g}$, it is shown in relation to the sampling rate. In the figure, it can be observed that the results based on the CP method are highly influenced by the number of oil drops present. Without any oil drop, the average error is of the order of magnitude of $10^{-4}$, but the presence of one oil drop increases this error up to the order of magnitude of $10^{-2}$. The presence of the second oil drop keeps increasing the value of the average error. Finally, no difference between the results related to the position of the second drop (in the contiguous slot or in the opposite one to the first) is shown. Since the $\mathrm{CP}$ method just counts pulses through the time series, the final result is not conditioned by the position of the second drop in relation to the first one. Finally, the effect of oil drop in the slotted wheel seems to be reduced in calibrations based on the FFT procedure.

As seen in Figs. 7-10, the residuals and the velocity errors are logically higher for small sampling frequencies than for higher sampling frequencies. In the present work, the stability condition of these variables with regard to the sampling rate has been considered to be reached when the value of the selected variable $\left(R_{S}\right.$ and $\left.\Delta V_{\text {avg }}\right)$ lies within the $\pm 5 \%$ of the average value from the 5 highest sampling rates (that is, from $1000 \mathrm{~Hz}$ to $25000 \mathrm{~Hz}$, both included), which are considered the most stable ones. In Fig. 11, the value of the sampling rate at which the stability condition is reached, SSR, is displayed in relation to the position of the second drop with regard to the first one.

The $R_{S}$ and $\Delta V_{\text {avg }}$ stabilization sampling rates related to the $\mathrm{CP}$ method does not depend on the position of the second drop in the slotted wheel of the opto-electronic system. In fact, the stabilization sampling rate of the residuals, $R_{S}$, is not affected by the presence of any oil drop. The velocity error, $\Delta V_{\text {avg }}$, is stabilized at a lower sampling rate when the drops are present in the slotted wheel, as the velocity error is highly increased with the presence of these drops (see Fig. 10, top). Therefore, the sampling rate at which the error is close to the final value is reached earlier.

On the other hand, the residuals, $R_{S}$, stabilization sampling rates from the calibrations based on the FFT procedure seem not to be very sensitive to the presence of one oil drop, having the same values shown by the calibrations based on CP. Only at some positions of the second oil drop the stabilization frequency slightly changes. With regard to the velocity error, $\Delta V_{a v g}$, the calibrations based on the FFT procedure seem to have a different stabilization sampling rate depending on the presence of a second oil drop. This variation between $892.86 \mathrm{~Hz}$ and 

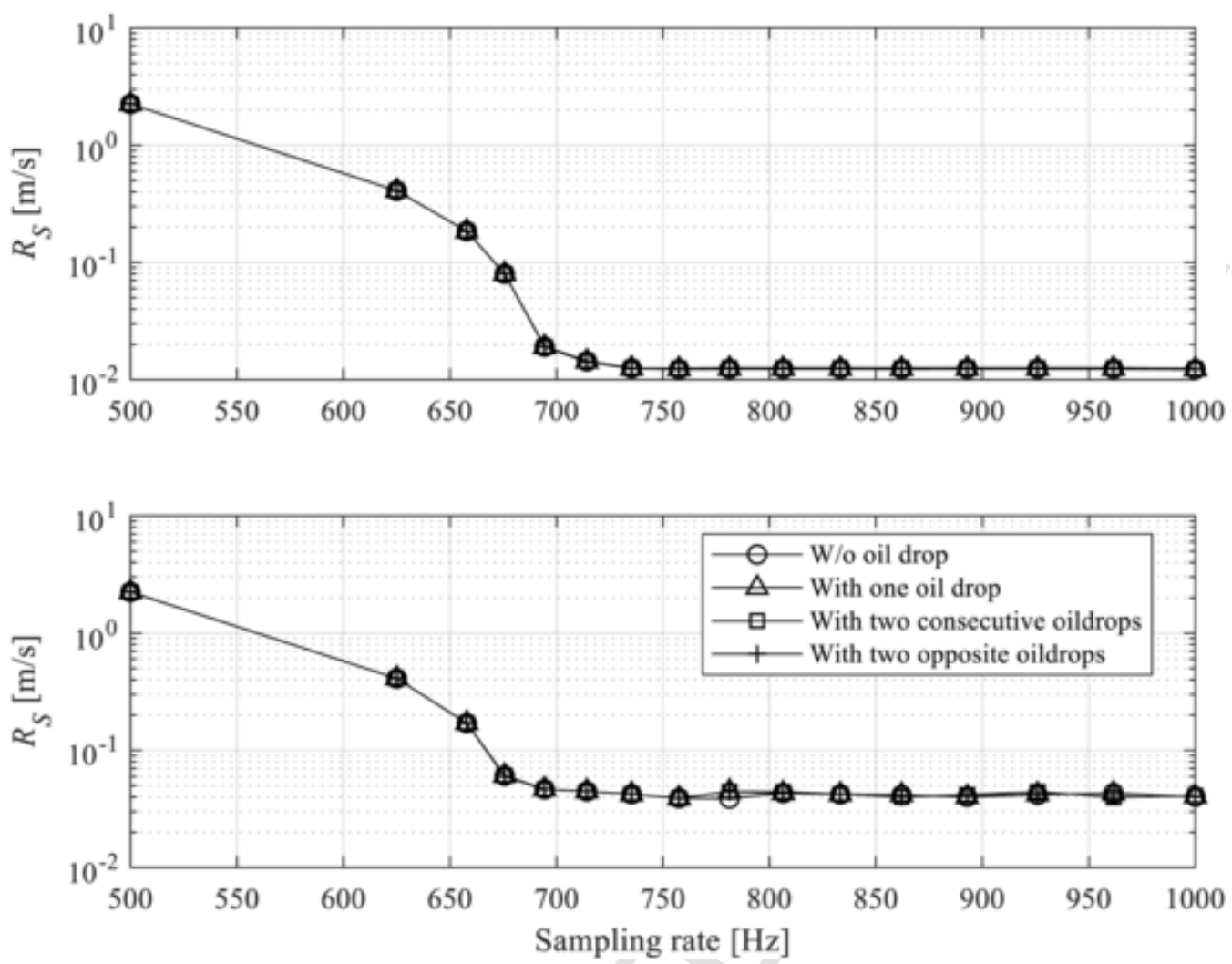

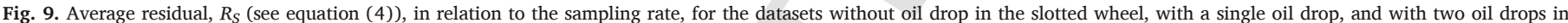

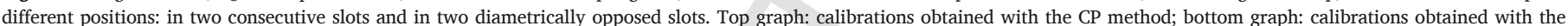
FFT method.

$1000 \mathrm{~Hz}$ of the stabilization sampling rate can be explained as the velocity error, $\Delta V_{a v g}$, shows some fluctuations in relation to the sampling rate (see Fig. 10, bottom).

The standard deviation of $R_{S}$ and $\Delta V_{\text {avg }}$ after SSR (that is, once the stabilization sampling rate has been reached) is displayed in Fig. 12 . Calibrations based on CP show very low values of this deviation. Only when the second oil drop is added, the standard deviation of the velocity error, $\Delta V_{\text {avg, }}$, slightly increases.

As for the calibrations based on the FFT procedure, the standard deviation of the residuals, $R_{S}$, is very similar for the configurations without oil drop and with one oil drop. When a second drop is added the standard deviation depends on the position of the second drop, but with no clear trend. Compared to the calibrations based on the CP procedure, the standard deviation of the residuals from the calibration based on the FFT procedure is larger. Regarding the standard deviation of the velocity error, $\Delta V_{a v g}$, the presence of the first oil drop makes it fall to a very low value. The second drop, however, changes this value for some of the positions of the oil drop.

\section{Conclusions}

The possible presence of oil drops in the slotted wheel of a cup anemometer opto-electronic system can lead to measurement errors, depending on the method used to process the output frequency of this wind sensor. Counting Pulses for extracting the output frequency (CP method) has a better performance than extracting the cup anemometers output frequency by using the Fast Fourier Transform (FFT), in both, residuals and velocity errors, when there is no oil drop. However, the presence of oil drops significantly increases the velocity error for the $\mathrm{CP}$ method, whilst the behavior for the FFT method remains unchanged.
If a cup anemometer is affected by dirt in the slotted wheel of the opto-electronic system, the observed errors in terms of measured wind speed can be quite large depending on the methodology used to extract the output frequency. This fact highlights the relevance of the present work as:

- dirt (oil drops from bearings lubrication) has proven to affect the aforementioned slotted wheel of anemometers, and

-both studied methodologies for output frequency extraction, CP and FFT, are commonly used in research and data processing within wind energy and meteorology sectors.

\section{Acknowledgments}

The authors are indebted to Prof. Ángel Sanz-Andrés for his constant support regarding the research program on cup anemometers performance. Besides, the authors are indebted to the LAC-IDR/UPM staff for their support in relation to the testing procedures. The authors are also grateful to Anna María Ballester Bohn for her kind help in improving the style of the text. Finally, the authors are truly grateful to the reviewers for their comments and suggestions that helped us to improve this work. 

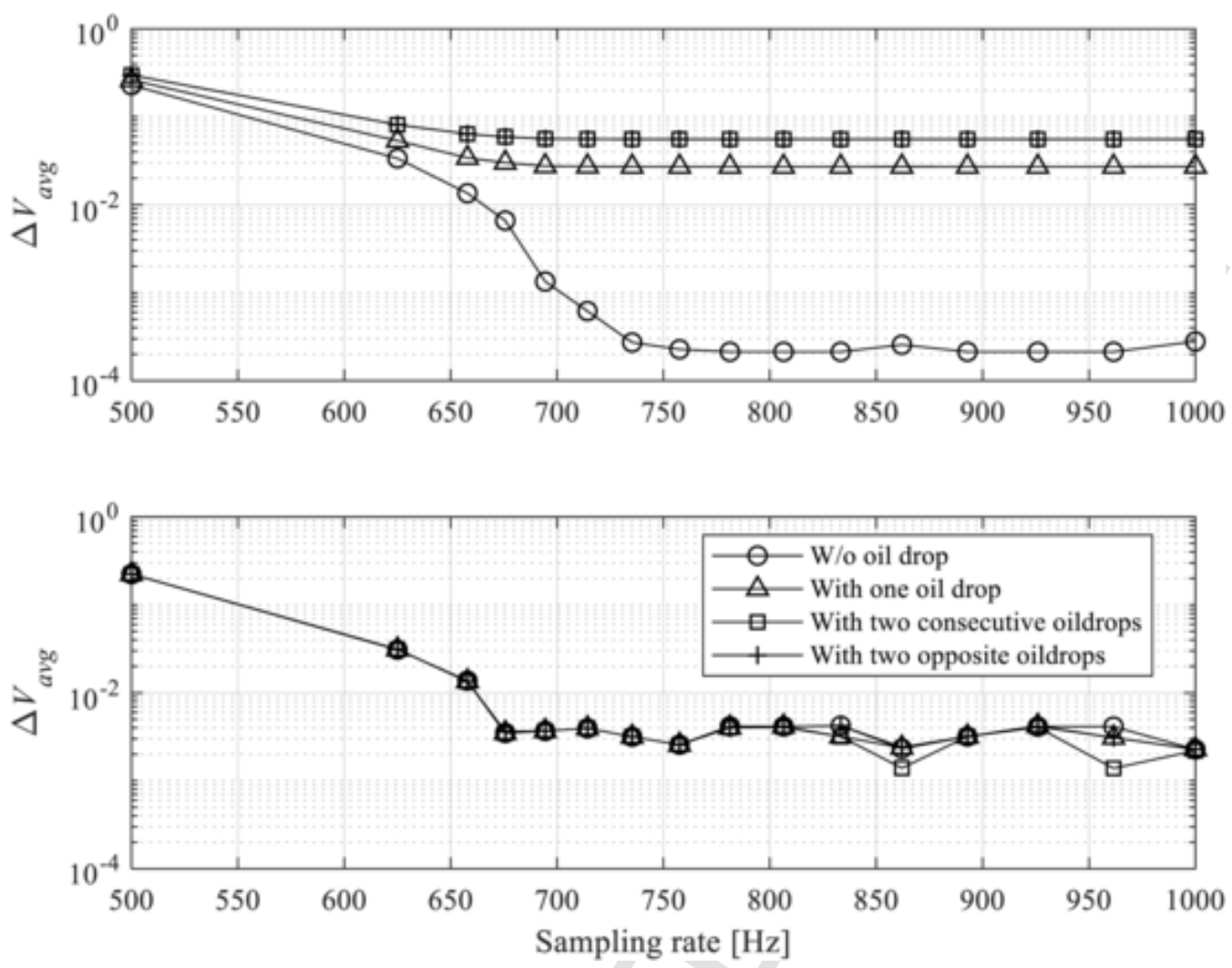

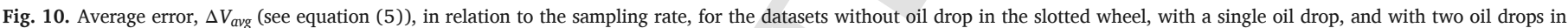

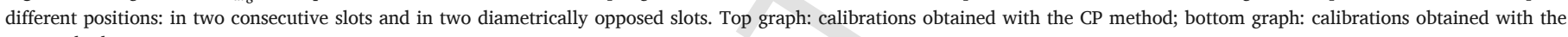
FFT method. 

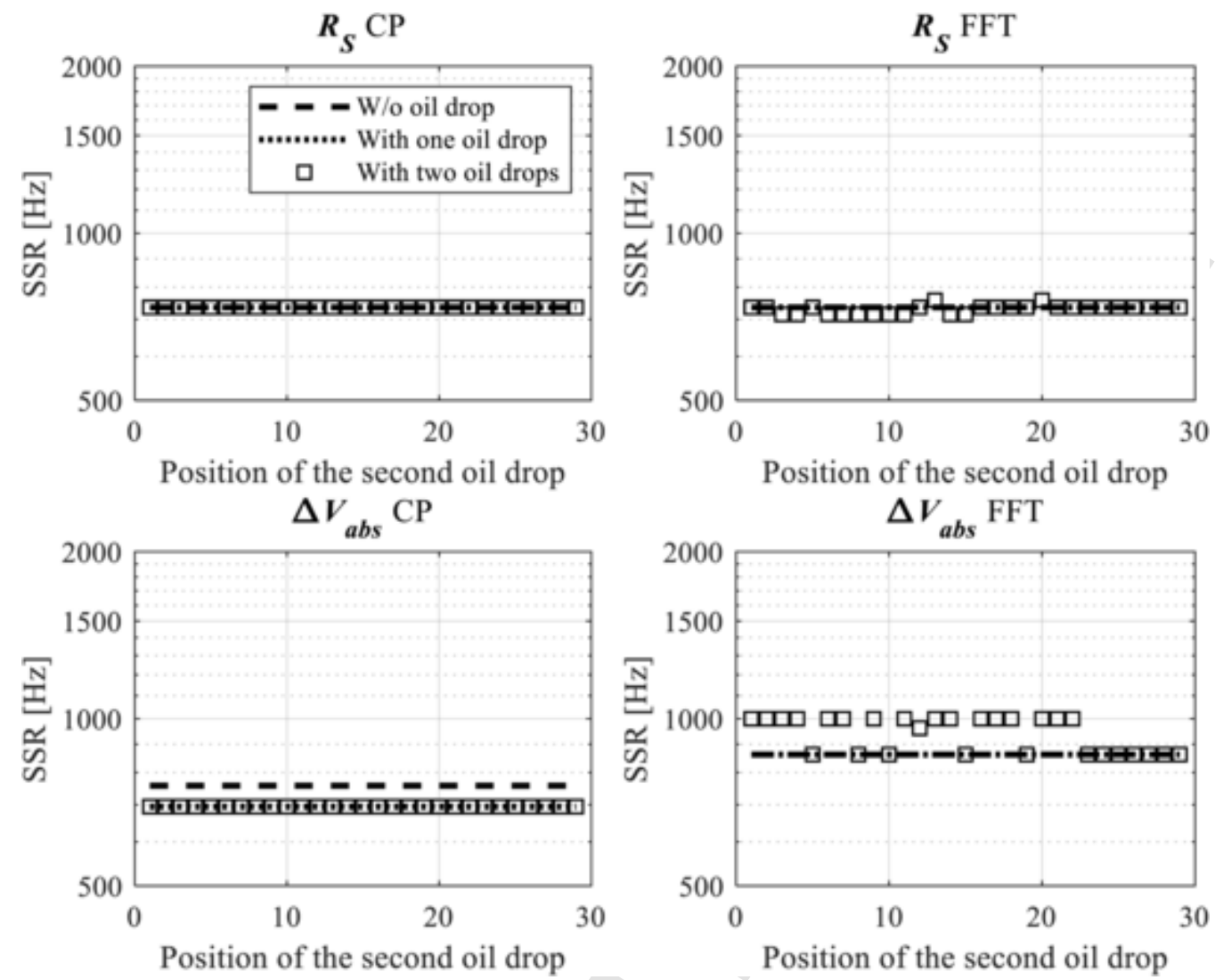

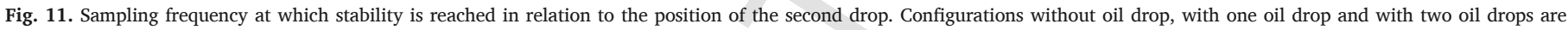
displayed. 

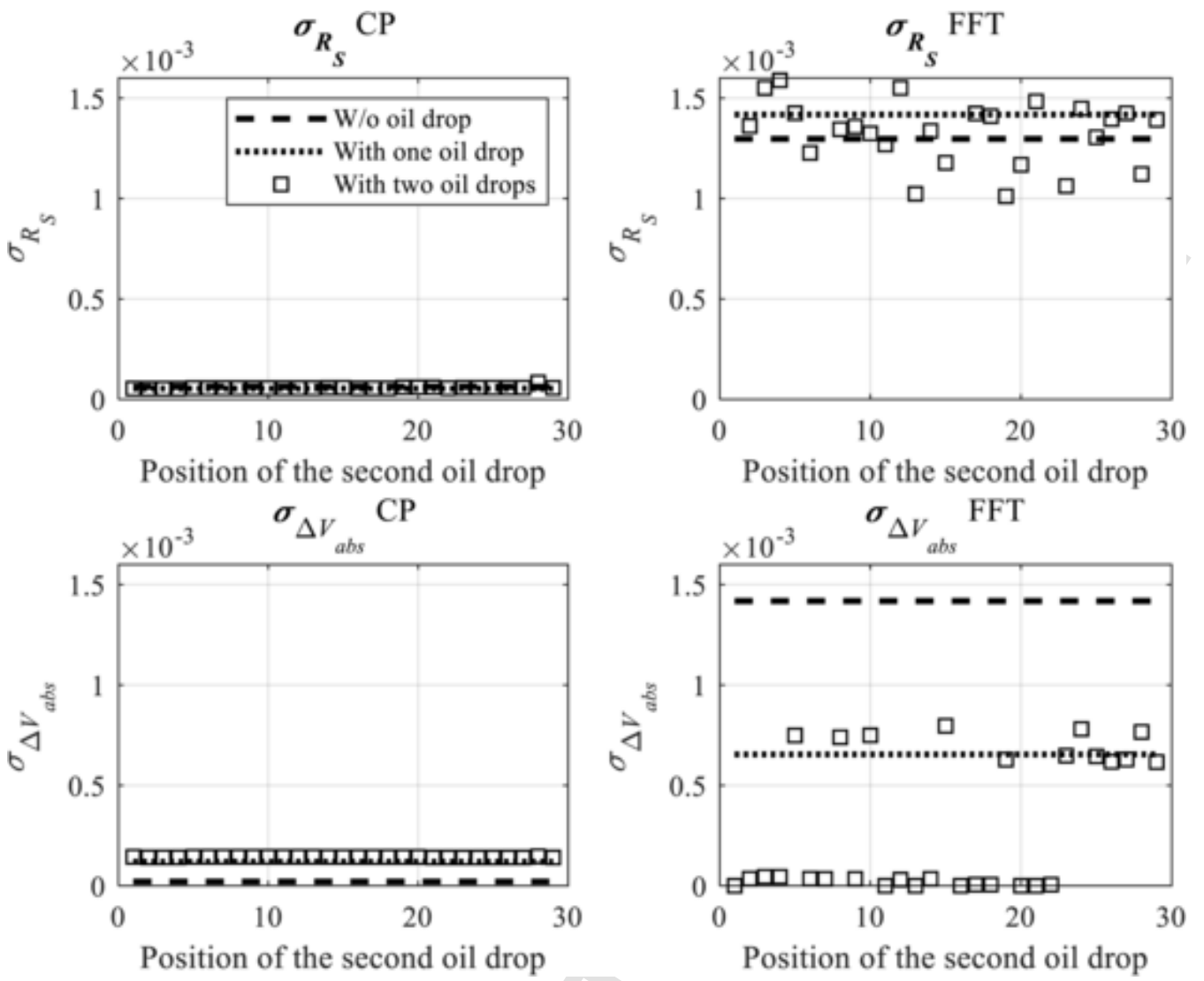

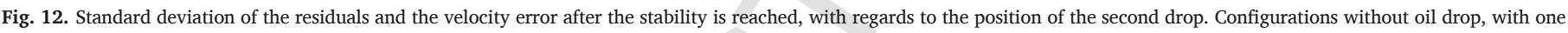
oil drop and with two oil drops are displayed.

\section{References}

[1] N. Stefanatos, P. Papadopoulos, E. Binopoulos, A. Kostakos, G. Spyridakis, Effects of long term operation on the performance characteristics of cup anemometers, Eur. Wind Energy Conf. Exhib. EWEC 2007, 7-10 May, Milan, Italy, 2007, pp. 1-6.

[2] A. Vidal-Pardo, S. Pindado, Design and development of a 5-channel arduino-based data acquisition system (ABDAS) for experimental aerodynamics research, Sensors 18 (2018) 1-20.

[3] S. Pindado, E. Vega, A. Martínez, E. Meseguer, S. Franchini, I. Pérez, Analysis of calibration results from cup and propeller anemometers. Influence on wind turbine Annual Energy Production (AEP) calculations, Wind Energy 14 (2011) 119-132.

[4] S. Pindado, A. Barrero-Gil, A. Sanz, Cup anemometers' loss of performance due to ageing processes, and its effect on annual energy production (AEP) estimates, Energies 5 (2012) 1664-1685.
[5] S. Pindado, A. Sanz, A. Wery, Deviation of cup and propeller anemometer calibration results with air density, Energies 5 (2012) 683-701.

[6] S. Pindado, J. Pérez, S. Avila-Sanchez, On cup anemometer rotor aerodynamics, Sensors 12 (2012) 6198-6217.

[7] E. Roibas-Millan, J. Cubas, S. Pindado, Studies on cup anemometer performances carried out at IDR/UPM Institute. Past and present research, Energies 10 (2017) 1-17.

[8] MEASNET, Cup Anemometer Calibration Procedure, 1997 (September 1997, updated 24/11/2008), Madrid, Spain. Version 1.

[9] MEASNET, Anemometer Calibration Procedure, 2009 (October 2009), Madrid, Spain. Version 2.

[10] A. Ramos-Cenzano, M. Ogueta-Gutierrez, S. Pindado, On the output frequency measurement within cup anemometer calibrations, Measurement 136 (2019) 718-723. 